e-migrinter

e-Migrinter

$18 \mid 2019$

L'ethnographie en migration(s)

\title{
Les migrants nigériens de retour de Libye
}

Ethnographie entre instabilité, précarité et honnêteté

Oriol Puig

\section{CpenEdition}

Journals

Édition électronique

URL : https://journals.openedition.org/e-migrinter/1721

DOI : 10.4000/e-migrinter.1721

ISSN : 1961-9685

Éditeur

UMR 7301 - Migrinter

Référence électronique

Oriol Puig, "Les migrants nigériens de retour de Libye », e-Migrinter [En ligne], 18 | 2019, mis en ligne le 12 septembre 2019, consulté le 20 mai 2021. URL : http://journals.openedition.org/e-migrinter/1721 ; DOI : https://doi.org/10.4000/e-migrinter.1721

Ce document a été généré automatiquement le 20 mai 2021.

Tous droits réservés 


\section{Les migrants nigériens de retour de Libye}

Ethnographie entre instabilité, précarité et honnêteté

Oriol Puig

\section{La Libye, Eldorado africain}

1 Selon le dernier rapport des Nations Unies sur les migrations internationales (2017), les migrations intra-africaines surpassent les flux migratoires dirigés vers l'Europe. L'Agence des Nations Unies pour l'Alimentation et l'Agriculture (FAO) et le Centre de Recherche Agricole pour le Développement (CIRAD) assurent que $75 \%$ des citoyens de l'Afrique sub-saharienne émigrent vers des pays voisins (Mercandalli ; Losch, 2017). De la même façon, 88,4 \% des expériences de migration en Afrique occidentale se réalisent dans la même région (Mercandalli ; Losch, 2017). Au croisement de ces dynamiques, et dès la deuxième moitié du XXème siècle, la Libye s'érige comme un Eldorado africain pour des milliers de personnes et ceci à cause de la découverte de gisements d'hydrocarbures en 1959 et la prise du pouvoir par Mouammar Kadhafi en 1969 (Haimzadeh, 2011). Les besoins en main d'œuvre étrangère - la Libye est le pays le moins peuplé d'Afrique, où seuls quelques 6 millions d'habitants résident - et le panafricanisme de Kadhafi forment un point d'inflexion des mobilités ouest-africaines, auparavant dirigées vers le sud, et dès lors reconduites vers le nord (Bensaâd, 2012). Avec une rhétorique "universaliste, anti-occidentale et révolutionnaire », le Guide ${ }^{1}$ utilise la migration comme une "arme diplomatique» pour se positionner entre le monde arabe, africain et européen (Staub, 2006).

2 Ainsi, des jeunes hommes d'entre 20 et 35 ans originaires du Niger, pays voisin de la Lybie et parmi les plus pauvres du monde (PNUD, 2018), prennent le chemin de la Libye au cours des années 1970 et 1980. Les premiers migrants sont des membres de l'ethnie Touareg, qui poursuivent une tradition nomade et migrent à la suite de graves épisodes de sécheresse dans le Sahel (Claudot-Hawad; Hawad, 1996). Parmi ces personnes, quelques-unes intègrent les rangs de la Légion Islamique de Kadhafi, alors que d'autres 
viennent essentiellement pour travailler. Suivent ensuite des jeunes membres des groupes Toubous, Kanouris et Haoussas du nord-est du Niger qui parviennent jusqu'à la région du Fezzan, au sud de la Libye, ce passage étant facilité par des liens culturels et commerciaux frontaliers anciens (Clanet, 1981). Enfin, notre ethnographie révèle des mobilités urbaines ayant pour origine la zone Sonray-Zarma ${ }^{2}$, commençant à la fin des années 1990 jusqu'au début des années 2000, distinctes de celles originaires du milieu rural, et qui impliquent un changement du modèle migratoire du sud vers le nord.

Ces déplacements s'édifient sur l'imaginaire que l'expression haoussa résume par Libya Kaman Turaï [La Libye comme l'Europe], et qui signifie que la Libye, avec l'indice de développement humain le plus élevé de la région, peut rivaliser avec l'Union européenne en termes de "modernité, justice sociale et égalité » (Rifkin, 2004). Mais, le conflit et la chute de Kadhafi en 2011 bouleversent ces mobilités circulaires transsahariennes et entrainent le rapatriement d'un million de Sub-sahariens vers leur pays d'origine, dont environ 300000 nigériens, selon les ONG internationales et locales. La xénophobie envers les noirs africains, déjà ancrée dans la société libyenne, s'accentue et la plupart des migrants persécutés sont accusés d'être des « mercenaires » du régime de Kadhafi ${ }^{3}$. Notre recherche se concentre sur les conséquences sociales, collectives et individuelles, de ces « retours involontaires $»^{4}$ ou forcés (Kleist, 2017) de milliers de personnes à Niamey. La capitale du Niger est moins affectée que les régions d'Agadez, Tahoua, Tillabéry ou Maradi ${ }^{5}$ (Mounkaila, 2015), elle représente cependant un terrain clé pour l'étude de ces retours.

4 L'article met en avant la méthodologie de notre étude en empruntant un virage réflexif vers une recherche honnête, empathique et engagée (Guber, 1991). Notre propos n'est donc pas de taire les difficultés et les obstacles rencontrés au cours de l'enquête. Bien au contraire, nous cherchons ici à mettre en lumière les opportunités que peut nous offrir un terrain alors même que l'enquête subissait les conséquences des contraintes sécuritaires, de l'instabilité dans la région et des limitations financières, la cantonnant à la capitale. L'enjeu est de revenir sur notre expérience ethnographique avec la volonté de répondre aux questions et aux doutes qui peuvent traverser les étudiant.es dans les prémisses de leur recherche, surtout si celle-ci, comme ce fut notre cas, se déroule dans une région du monde affectée par des conflits et/ou se réalise dans des conditions financières difficiles comme c'est de plus en plus le cas pour les chercheur.es en sciences sociales.

\section{Contourner les obstacles liés à l'instabilité de la région}

5 Nos travaux de recherche sont conduits dans le milieu urbain de Niamey, et durant plusieurs périodes suivant la chute de Kadhafi, entre 2012 et 2016. En acceptant que " toute démarcation d'un phénomène sous étude est artificielle " et n'importe quelle limite espace/temps « est conventionnelle et, dans quelque mesure, arbitraire ", selon Krotz (1991), notre décision s'assume "partielle», basée sur des aspects à la fois subjectifs et objectifs, détaillés ci-après.

6 Notre idée initiale était de nous baser à Agadez, principal carrefour africain, tourné vers le nord, et où circulent divers types de marchandises - tabac, drogues, armes... ainsi que des personnes (Brachet, 2009). Néanmoins, les contraintes sécuritaires nous ont dissuadé de mener l'enquête dans cette région. Les fréquentes attaques attribuées aux groupes djihadistes et les enlèvements de ressortissants européens ont entrainé le 
décret par la communauté internationale de fortes mesures de sécurité pour empêcher l'accès au nord du Niger et du Mali ${ }^{6}$ (Shaw, 2013). A la même période, les violences perpétrées par le groupe Boko Haram au sud-est du Niger ${ }^{7}$ et la "guerre contre le terrorisme " initiée par le Président nigérien, Mahamadou Issoufou, ont dégradé la situation sociale et économique du pays, déjà fragile, et marquée par des crises alimentaires structurelles (entre 4 et 5 millions de personnes en risque d'insécurité alimentaire, selon les Nations Unies).

Dans ces circonstances et à cause de craintes personnelles induites par les recommandations des annassaras ${ }^{8}$ mais aussi par de nombreux informateurs locaux, nous avons changé finalement l'espace socioculturel à étudier, en priorisant Niamey au lieu d'Agadez'. Nous sommes conscients du privilège qui nous permet, en tant que chercheur, de changer de terrain, alors que les migrants ne pourraient pas changer leur projet de retour si facilement (Dujmovic, 2016). Mais en usant de ce privilège, nous avons révélé l'importance d'un groupe d'individus de retour à la capitale, groupe généralement ignoré à l'époque par les organismes humanitaires et la production scientifique.

8 Le fait de travailler à la capitale a facilité la «familiarisation », dont parle Malinowski (1989), avec le groupe étudié, car nous avons profité des relations préalablement forgées lors de séjours précédents ${ }^{10}$. De la même manière, la possibilité de travailler avec des personnes issues d'un milieu urbain, ayant une certaine maîtrise de la langue française, un taux d'alphabétisation plus élevé et, en général, un accès majeur à la « modernité $»^{11}$ (Alzouma, 2015), a rendu plus abordable le travail de terrain. Cette enquête a fourni des informations indispensables pour comparer la production de l'altérité par les migrants, selon leurs origines urbaines et rurales. Nous avons observé des différences concernant le type de travail, la sociabilité et les attentes associées au projet migratoire, au départ comme au retour. Ainsi, comme l'affirme Mahamadou, Eux (les « ruraux»), ils ont un projet de vie entièrement différent. Ils restent moins longtemps, gagnent de l'argent et le dépensent. Nous, en revanche, nous souhaitons l'investir pour en faire quelque chose. Nous sommes des entrepreneurs.

Que ce soit un retour en zone rurale ou en zone urbaine, il s'agit essentiellement de mobilités circulaires. Cependant, nous observons que la migration urbaine se conçoit à plus long terme que la migration rurale - cette dernière durant entre 3 et 5 ans - et se perçoit comme une "opportunité » de progrès avec un degré plus élevé d'individualisation en comparaison avec les déplacements des travailleurs agricoles, fondés sur un caractère saisonnier et davantage déterminés par le climat, la précarité et la communauté. Nous voyons déjà émerger une première hypothèse, dont le « développement » est lié à un problème d'ordre méthodologique : le contournement des obstacles sécuritaires et politiques a facilité l'observation d'un phénomène original, ayant lieu en milieu urbain plutôt que dans les campagnes plus étudiées.

\section{Faire face à la précarité}

Notre travail a été conditionné par la contrainte budgétaire. La précarité des chercheurs en sciences sociales est de notoriété publique dans de nombreux pays, mais elle l'est d'une manière préoccupante en Espagne, en conséquence des plans d'austérité décrétés par les gouvernements conservateurs de Mariano Rajoy (2011-2018). Ces derniers ont entrainé la diminution du budget public dédié à la recherche, et cette 
réduction drastique a encore plus affectée les sciences sociales, souvent victimes d'un préjugé idéologique plus fort que les sciences exactes dans un contexte général de mercantilisation de l'académie (Lamy, 2006; Mihalache, 2018). En outre, l'absence totale de la zone du Sahel dans l'imaginaire espagnol - lors que la France conserve encore des intérêts politiques et économiques dans la région, due à son histoire coloniale - et l'approche critique de notre recherche envers les politiques européennes de restriction migratoire et d'externalisation des frontières ${ }^{12}$, surtout consacrées à la "gestion des flux migratoires ", ont rendu plus difficile encore l'accès au financement (Hamadou, 2018).

11 De par ces contraintes, le travail de terrain a eu lieu entre 2012 et 2016 pendant divers voyages de plusieurs mois chacun. Ces périodes cumulées - d'une durée totale d'une année - nous ont permises d'analyser l'évolution temporelle de la thématique abordée. En effet, nous avons mené une étude longitudinale des migrants nigériens dans leur retour au pays. Au fil de ces périodes sur place, mais aussi via l'utilisation des réseaux sociaux comme Facebook ou Whatsapp, grâce auxquels nous avons suivi leurs traces et maintenu la communication, nous avons posé la question des reconfigurations identitaires opérées dans le groupe à plusieurs temps de leur réinstallation, de leurs premiers pas à leur revendication d'intégration dans leur propre pays.

Notre ethnographie s'est basée sur des techniques qualitatives associant l'observation participante et des conversations informelles, principalement dans les fadas, ces espaces primordiaux de sociabilité masculine au Niger. Ces rassemblements d'hommes "d'âge relativement proche » regroupés «sur la base de l'affinité, de l'interconnaissance acquise par la fréquentation d'un même établissement, par le voisinage, ... » (Boyer, 2017), entraînent une appropriation de l'espace public au-delà de l'ethnie, la classe ou l'âge, même si ces aspects jouent aussi un rôle.

Il y a les fadas de fonctionnaires, de commerçants, de travailleurs, de chômeurs, ... On s'assoit, on prend le thé, on s'amuse, on discute, on se protège, explique un ancien migrant à Niamey (propos recueillis le 14/03/2016).

Dans le cas des personnes de retour de Libye, les fadas impliquent une socialisation par des expériences communes.

On s'est connu en Libye et si Kadhafi était vivant, chacun serait de son côté. On est revenu et on s'est soutenu. C'est une des fadas des exodants, explique Abdelaziz (propos recueillis à la Fada dite Poudrière, 29/02/2016).

Pour cette raison, notre recherche s'est déroulée dans ces espaces dans les quartiers où les personnes de retour y résident majoritairement, surtout la commune I, III et IV. 


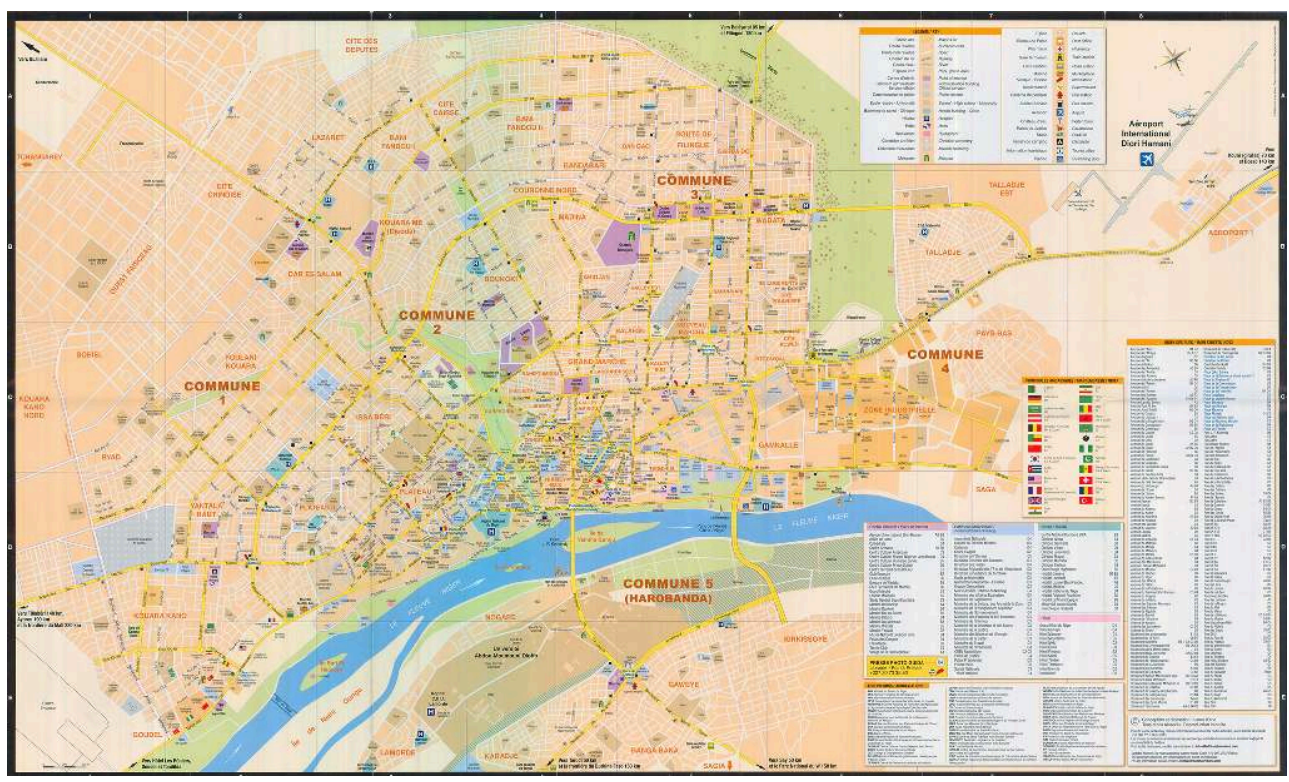

Source : Éditions Laure Kane, France (2015).

De cette manière, nous avons priorisé la valeur ethnographique de ces regroupements par rapport à l'organisation artificielle de focus-groups, créés par des initiatives " développementalistes » (Olivier de Sardan, 1995). Nous avons pu analyser ces espaces « naturels » et même d'y établir des relations personnelles au travers desquelles nous avons mené des entretiens personnels, à la maison ou au travail. En fait, la fada a été le principal espace d'étude et la porte d'entrée dans le collectif, même si nous avons aussi mené des entretiens individuels. Les entretiens individuels ont permis de traiter de sujets difficiles à aborder au niveau collectif, comme par exemple, les épisodes d'incarcération de quelques-uns ou encore, les relations de pouvoir existants entre les différents membres de la fada. Ainsi, nous avons identifié plusieurs fadas de migrants de retour, et nous avons échangé aussi avec le Collectif des Rapatriés Nigériens (CORNI), l'organisation auto dénommée porte-parole de la cause générale des retournés. Les rencontres aux fadas consistaient en des conversations avec des hommes autour d'un verre de thé, la principale boisson de socialisation. En ce sens, nous avons passé «le plus de temps possible et le plus de moments d'une certaine quotidienneté », selon Guber (1991), avec des personnes de retour, soit rapatriées par l'Organisation Internationale des Migrations (OIM) ou bien rentrés de leurs propres moyens. L'ethnographie s'est étendue aux familles plus larges, et nous avons pu entamer un dialogue avec des parents, amis et des associations liées à la mobilité. Nous avons également réalisé des entretiens semi-structurés auprès d'autorités, agences et ONG internationales et locales comme Médecins Sans Frontières, le Comité Internationale de la Croix Rouge (CICR) ou le Comité Ad-hoc pour les Rapatriés du Gouvernement nigérien, entre autres, qui traitaient autant le collectif que les bénéficiaires individuels de leurs services. En ce sens, le plongement dans les fadas nous a servi à analyser leur contexte au-delà d'une perspective "victimiste » et verticale de ces personnes, en priorisant donc, un regard plus holistique de leurs circonstances. 


\section{Négocier une position de «blanc dominant »}

Depuis le début de cet article, nous avons mis en exergue les défis épistémologiques liés à la perception de l'homme "blanc dominant " que nous sommes, elle-même liée à "l'hégémonie culturelle et la supériorité politique et économique ", attachée à la figure du colonisateur, selon Mbongo (2003). Nous avons dû admettre que

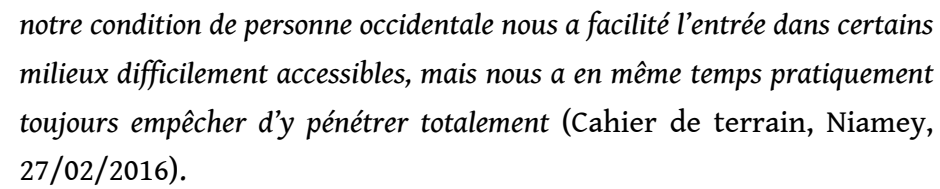

Ainsi, notre condition d'homme blanc nous a permis d'observer des coutumes et habitudes sociales, mais souvent depuis une perspective privilégiée. Les seuls moments où nous avons sentis une certaine égalité entre nous et nos interlocuteurs étaient lorsque quelqu'un s'adressait à nous avec des expressions telles que «tu es devenu nigérien » ou boro bi - noir, autochtone - annassara ${ }^{13}$ (Lévi-Strauss, 1983). Nous avons pris conscience de la difficulté de dépasser la distance culturelle existante, et, pour la réduire, la première exigence a été de la reconnaître. Une fois cette distance reconnue et acceptée, nous nous sommes adaptés en mettant en place des stratégies de rapprochement comme une écoute active, une volonté d'apprendre et une empathie. Même si nous connaissons la méfiance de Lévi-Strauss envers le concept d' "empathie " en ethnographie, nous l'utilisons ici en tant que « reconnaissance de la subjectivité du chercheur dans le processus de connaissance et du champ des significations sociales [...] dans lequel le chercheur doit reconstruire la logique et la cohérence propres et inhérents à la culture étudiée " (Guber, 1991). En ce sens, nous avons abordé le travail de terrain comme une «expérience unique» (Lévi Strauss, 1958); une "école d'humilité ", pour reprendre les mots de Denise Paulme, et une "négociation et construction de mon propre je » (Geertz, 1989).

16 À cet égard, nous avons déconstruit notre bagage personnel et culturel en cherchant à être honnête avec les informateurs, avec nous-même et en revendiquant notre subjectivité comme valeur scientifique. Nous l'avons incorporé « pas sur une abdication de l'exigence scientifique de l'objectivité, [mais bien sur une] reconnaissance des conditions objectives d'intersubjectivité dans les relations du champ et de l'interprétation scientifique", selon les mots de Florence Weber (2009). De cette manière, nous avons pensé indispensable de dévoiler notre cosmovision et condition sans «une prétentieuse humilité herméneutique vers l'Autre, imprégnée de narcissisme et d'auto-adoration", comme le considère Llobera (1990), mais plutôt comme un comportement nécessaire pour l'obtention d'une connaissance rigoureuse. Ainsi, notre rang d'homme blanc, jeune, européen et descendant de migrants andalous en Catalogne, une petite nation de l'État espagnol, a sans aucun doute contribué à forger notre regard sur notre terrain. Nous savons que le fait d'être un homme dans une société androcentrique nous a certainement facilité l'accès aux fadas, mais nous a en même temps rendu plus difficile l'accès aux femmes, car les épouses ont besoin de l'autorisation du mari pour parler avec d'autres hommes au Niger. C'est pour cela que l'accès aux foyandis, l'équivalent féminine des fadas a été ardu, mais notre insistance nous a permis finalement d'échanger avec quelques femmes de migrants retournés, ce 
qui nous a fourni des témoignages intéressants sur le mariage ou sur leurs séjours en Libye.

Par ailleurs, les a priori et les projections liées à ma couleur de peau blanche pouvaient créer des attentes au sein de ce collectif qui se présentait lui-même comme «vulnérable» du fait du retour forcé. Pour cette raison, et ce dès les premières rencontres, nous avons expliqué quel était notre objectif académique, sans nourrir de faux espoirs économiques ou matériels. Nous avons également explicité la différence entre les techniques d'observation participante et de vision observante, insistant sur le fait que nous ne serions là que pour observer et non pas participer. De fait, nous avons décidé, après beaucoup de tensions internes, de finalement prioriser l'observation au lieu de l'action, en nous positionnant dans la lignée d'auteurs comme Kabunda, Sodupe et Moure (2009), qui clament que "l'autorité africaine ne se légitime pas avec l'intervention, sinon simplement en étant, sachant être dans le centre même de la quotidienneté de ces gens ». Grâce à cette posture, notre ethnographie réflexive, mais pas collaborative, n'a pas renoncé à l'engagement éthique et moral, comme l'affirme Katzer et Samprón (2011) ; et a toujours eu comme ambition de contribuer à rendre visible le message du collectif retourné. Nous avons regroupé leurs revendications, sans pour autant les mettre au centre de la structure et conceptualisation de notre travail, mais en espérant que nos conclusions puissent servir à la libre circulation, déjà reconnue par le protocole spécifique de la CEDEAO, mais aussi défendue comme droit humain par plusieurs organismes et entités sociales au niveau mondial. C'est le cas du Groupe d'Information et de Soutien des Immigrés (GISTI) par exemple, mais autant d'autres qui considèrent le droit à émigrer consacré dans la Déclaration des droits humains ${ }^{14}$. En acceptant donc "les responsabilités morales et politiques de nos actions ", comme l'affirme Sánchez Carretero (2003), nous avons articulé une recherche positionnée en faveur des personnes en mouvement, en s'éloignant d'une posture de vulnérabilisation des migrants et des discours simplistes et réducteurs basés exclusivement sur des facteurs push-pull qui mettent en exergue la relation entre manque de développement et migration (Akesson; Baaz, 2015).

\section{Honnêteté et rigueur : orgueil migrant}

18 En ce sens, sans dissocier notre bagage culturel et social de notre vision de chercheur, nous nous sommes approchés des mobilités transsahariennes en tant que petit fils de migrants andalous - région au sud de l'Espagne - déplacés en Catalogne au cours des années 1960 et 1970 à cause des crises politiques et économiques. Sans entrer ici en profondeur sur l'organisation territoriale de l'État espagnol, il est vrai que la condition de descendant de migrant en Catalogne, nous a aidés à être plus sensible à la diversité culturelle et sociale nigérienne. C'est ainsi que nous avons tenu à souligner la flexibilité identitaire dans ce contexte africain à plusieurs niveaux, nationale, ethnique, régional ou religieux. D'un autre côté, la recherche nous a permis aussi de rétablir notre conscience de classe, depuis longtemps oubliée par notre famille, et de nous réaffirmer dans un " orgueilleux migrant ", compris comme une « cicatrice, marque de l'étranger, stigmatisation morale qui ne guérit pas ", d'après Sennett (2014).

19 Ainsi, nous avons analysé le discours ambivalent des personnes retournées, entre « victimisme » et exaltation de leur rang voyageur, qui se définissent comme un groupe singulier; et nous avons découvert un collectif caractérisé par des apprentissages 
obtenus hors du pays, ce que Levitt (2007) dénomme comme des «transferts sociaux ». Les personnes retournées, de fait, admirent le système socio-économique libyen et méprisent le Niger, valorisant leur expérience migratoire grâce à laquelle ils ont obtenu des métiers techniques et des gros salaires. Rentrés au pays, ils sentent l'« échec » et la «frustration » du retour, et en même temps, ils doivent assumer la transformation de leur rôle social puisque, de "garants", ils deviennent "dépendants" de la famille (Puig, 2017). Ils remarquent une altérité avec leurs compatriotes qui n'ont jamais voyagé, en se sentant supérieurs; et, se revendiquent comme des victimes de guerre pour essayer d'accéder à la "rente de développement » dont parle Olivier de Sardan (1995), soit les subventions et aides économiques fournies par des organisations et ONG internationales, très présentes au Niger. Avec cet objectif, ils créent des organisations pour mettre en place des projets de développement auprès de l'OIM ou bien ils rentrent dans des partis politiques afin d'y chercher une rétribution (Blundo, Olivier de Sardan, 2007). Au bout du compte, ils concrétisent plusieurs stratégies de résilience pour réparer ce que Marie (1997) appelle une "dette communautaire", ou le lien indissociable entre communauté et individu. En quelque sorte, les personnes retournées essaient de rembourser les attentes que la communauté a eu envers eux au moment de leur départ, et qui n'ont pu être remplies lors d'un retour «les mains vides », et d'un séjour écourté par la guerre.

\section{Conclusion}

On peut donc conclure que les contraintes sécuritaires et financières nous ont aidé à concentrer nos efforts sur une thématique originale, caractérisée par des tensions et des complémentarités entre les individus et leur communauté, avec la mobilité au centre. La délimitation des marges du travail a facilité l'extraction de conclusions singulières sur le phénomène migratoire intra-africain, insuffisamment traité par le milieu académique. De cette manière, nous avons contribué à faire émerger l'explication d'un nouveau type de mobilité urbaine transsaharienne initiée dans les années 2000 suite à des crises économiques et politiques et à l'appauvrissement de la classe moyenne Sonray-Zarma, héritière de l'administration coloniale. Nous avons révélé que ces déplacements de personnes de la capitale vers la Libye correspondent à des logiques différentes par rapport aux projets des personnes rurales, même si tous prennent part à des migrations circulaires. À l'inverse, les mobilités rurales sont saisonnières, plus précaires et conditionnées pour la communauté, tandis que la migration urbaine est une initiative à plus long terme, entre trois à cinq ans, parfois plus, et se conçoit comme "opportunité» de progrès avec plus de marge d'individualisation.

Par ailleurs, les obstacles apparus du fait de l'instabilité et de la précarité financière nous ont permis d'observer l'évolution du groupe étudié lors de diverses étapes suivant leur retour. À cet égard, nous avons constaté la «frustration » du collectif retourné à Niamey dans les premiers moments du retour, pour ensuite remarquer la mise en place de diverses stratégies de résilience. Le travail en différentes périodes nous a aidé à montrer l'importance de l'appartenance urbaine ou rurale dans la configuration des projets migratoires, à travers l'identification ethno-régionalistes attachée à la zone d'origine, identification qui dépasse le concept d'ethnicité. D'un autre côté, nous avons constaté l'importance de l'« industrie du développement » (Olivier de Sardan, 1995) qui 
contribue à accentuer notre position de «blanc dominant », attribuée par la population de ce pays néocolonisé de fait - avec plus de $50 \%$ de son budget public dépendant de l'Aide Officielle au Développement.

Grâce à ces remises en question, nous avons confirmé notre posture "non participative" ni collaborative pour ne pas contribuer à créer une société plus dépendante. Cependant, nous n'avons pas renoncé à développer une ethnographie engagée pour le droit à la libre circulation, consacré par les droits humains et par plusieurs protocoles régionaux en Afrique de l'Ouest. Ainsi, sans renoncer à la subjectivité comme principe scientifique, nous avons matérialisé une ethnographie pendant des années, toujours conscients de notre responsabilité éthique et morale envers le collectif étudié et aussi envers la production académique. Pour cette raison, nous avons évoqué des aspects personnels indissociables de notre condition de chercheur pour essayer de réduire la distance avec nos informateurs, en revendiquant l'honnêteté et l'empathie comme principes réflexifs indispensables dans les études ethnographiques. De cette manière, en rejetant le simplisme et le paternalisme parfois, aussi, le racisme -, nous avons revendiqué notre " orgueil de migrant» et la récupération de notre conscience de classe longtemps oubliée ; et nous avons mené une lutte obstinée pour une ethnographie réflexive, rigoureuse et engagée dans des situations instables et précaires. En définitif, nous exigeons toujours une déconstruction de nos postures en tant que chercheurs ainsi que de nos thématiques abordées pour aider à dépasser une fois pour toutes les approches réductionnistes, intéressées et qui, en quelque sorte, violent les droits humains.

\section{BIBLIOGRAPHIE}

Akesson, Lisa Baaz, Maria Eriksson (2015) Africa's return migrants. The new developpers ?, Londres, Zed Books, 192 p. (Africa Now).

Alzouma, Gado (2015) The opportunity exists. Why don't they seize it ?' Political (In) Competence and the Potential of ICTs for Good Governance, Stability : International Journal of Security \& Development, $\mathrm{n}^{\mathrm{0}} 29$, pp. 1-17.

Bensaâd, Ali (dir.) (2012) La Libye révolutionnaire, Paris, Karthala, 245 p. (Politique Africaine, nº 125).

Blundo, Giorgio ; Olivier de Sardan, Jean Pierre (2007) État et corruption en Afrique. Une anthropologie comparative des relations entre fonctionnaires et usagers (Bénin, Niger, Sénégal), Paris, Karthala, 376 p.

Boyer, Florence (2017) «Faire fada' « : entre imaginaire de la révolte et désir d'autonomie? (Niamey), in Fouquet, T. ; Goerg, O. (dir.) Citadinités subalternes en Afrique, Paris, Karthala. Brachet, Julien (2009) Migrations transsahariennes : vers un désert cosmopolite et morcelé (Niger), Bellecombe-en-Bauges, Édition du Croquant, 321 p. 
Brachet, Julien (2018) Manufacturing smugglers : from irregular to clandestine mobility in the Sahara, Annals, $\mathrm{n}^{\circ}$ 676, pp. 16-35.

Chabal, Patrick ; Daloz, Jean-François (1999) L'Afrique est partie ! Du désordre comme instrument politique, Paris, Éditions Economica, 196 p.

Claudot-Hawad, Hélène ; Hawad, E. (dir.) (1996) Touaregs. Voix solitaires sous l'horizon confisqué, Paris, Ethnies, 255 p.

Clanet, Jean Charles (1981) L'émigration temporaire des Toubou du Kanem vers la Libye, Cahiers Géographiques de Rouen, nº 15, pp. 15-33.

Crespo, Rafael (2006) Rutas migratorias transaharianas : de los puertos caravaneros a los cayucos, Nova África, no 19, pp. 77-93.

Dujmovic, Morgane (2016) «Follow the plot, the thing, the people ». L'ethnographie multi-située pour saisir les territorialités du contrôle migratoire en Croatie, Journée de Doctorants Migrinter.

Geertz, Clifford (1988) L'anthropologue comme auteur, Paris, Métaillé, 152 p.

Guber, Rosana (1991) El salvaje metropolitano. Reconstrucción del conocimiento social en el trabajo de campo, Buenos Aires, Paidós, 323 p.

Haimzadeh, Patrick (2011) Au coeur de la Libye de Kadhafi, Paris, JC Lattès, 200 p.

Hamadou, Abdoulaye (2018) La gestion des flux migratoires au Niger entre engagement en contraintes, La revue des droits de l'homme, pp. 1-15.

Katzer, Leticia ; Samprón, Agustín (2012) El trabajo de campo como proceso. La "etnografia colaborativa" como perspectiva analítica, Revista Latinoamericana de Metodología de la Investigación Social, $\mathrm{n}^{\mathrm{o}} 2$, pp. 59-70.

Kleist, Nauja (2017) Disrupted migration projects : the moral economy of involuntary return to Ghana from Libya, Africa, no 87, pp. 322-342.

Krotz, Esteban (1991) Viaje, trabajo de campo y conocimiento antropológico, Alteridades, no 1 , pp. 50-57.

Lamy, Erwan ; Shinn, Terry (2006) L'autonomie scientifique face à la mercantilisation. Formes d'engagement entrepreneurial des chercheurs en France, Actes de la recherche en sciences sociales, no 164 , pp. 23-50.

Lecocq, Baz (2010) Disputed desert, Leiden, Brill, 434 p.

Lévi-Strauss, Claude (1983) Le Regard Éloigné, Paris, Plon, 398 p.

Lévi-Strauss, Claude (2003) Anthropologie structurale, Paris, Plon, 492 p.

Levitt, Peggy (1998) Social Remittances : Migration Driven Local-Level Forms of Cultural Diffusion, International Migration Review, vol. 32, ํํ 4, pp. 926- 948.

Llobera, Josep R. (1990) La identidad de la antropología. Barcelona, Buenos Aires, Anagrama, 160 p.

Malinowski, Bronislaw (1989) Les Argonautes du Pacifique occidental, Paris, Gallimard, 606 p.

Marie, Alain (1997) L'Afrique des individus. Itinéraires citadins dans l'Afrique contemporaine (Abidjan, Bamako, Dakar, Niamey), Paris, Karthala, 438 p.

Mercandalli, Sara ; Losch, Bruno (2017) Rural Africa in motion. Dynamics and drivers of migration South of the Sahara. Rome, FAO and CIRAD, $60 \mathrm{p}$. 
Mbongo, Nsame (2003) Identité et altérité en Afrique : étude de la contradiction autochtoneétranger, le cas de Douala, in Coquery-Vidrovitch, C. ; Goerg, O. ; Mandé, I. (dir.) Être étranger et migrante en Afrique au XXe siècle. Volume 2, Paris, L'Harmattan, pp 281-288.

Mihalache, Anca (2018) Sciences humaines ; les jeunes chercheurs à l'épreuve du néolibéralisme académique, Libération, [réf. du 13 janvier 2019] [Disponible sur Internet ]

Moisseron, Jean Yves ; Belalimat, Nadia (2012) L'après-Kadhafi : nouveaux défis en Libye et au Sahel, in Mokhefi, M. ; Antil, A. (dir.) Le Maghreb et son Sud : vers des liens renouvelés, Paris, CNRS Éditions, pp. 59-74.

Mounkaila, Harouna (2015) La gestion des rapatriés de Libye dans la commune de Tchintabaraden (Niger) : les défis de l'urgence et du durable, Afrique et développement, vol 40, no 1, pp. 99-117.

Nabara, Hamidou (2014) Migration de retour, stratégies de réinsertion et changement social dans la Commune urbaine de Tchintabaraden, Mémoire de Master, Niamey, Université Abdou Moumouni de Niamey.

Nations Unies (2017) International Migration Report, Highlights, New York, 38 p.

Olivier de Sardan, Jean Pierre (1995) Anthropologie et développement. Essai en socio-anthropologie du changement social, Paris, Karthala, $224 \mathrm{p}$.

Puig, Oriol (2017) Libya Kaman Turaï. El Dorado libio : los retornados nigerinos en Niamey, Thèse doctorat, Barcelona, $480 \mathrm{p}$.

PNUD (2018) Indices et indicateurs du développement humain : Mise à jour statistique 2018, Nueva York.

Rifkin, Jeremy (2004) The European Dream : How Europe's vision of the future is Quietly eclipsing the American Dream, Los Angeles : TarcherPerigee

Sánchez Carretero, Cristina (2003) Voces y escritura : La reflexividad en el texto etnográfico, Revista de Dialectología y Tradiciones populares, $\mathrm{n}^{\circ}$ 58, pp. 71-84.

Sennett, Richard (2014) El extranjero. Dos ensayos sobre el exilio, Barcelona, Anagrama, $136 \mathrm{p}$.

Shaw, Scott (2013) Fallout in the Sahel : The Geographic Spread of Conflict From Libya to Mali, Canadian Foreign Policy Journal, vol 19, pp. 199-210.

Sodupe, Kepa ; Kabunda, Mbuyi ; Moure ; Leire (2009) África subsahariana. Perspectivas sobre el subcontinente en un Mundo Global, Universidad País Vasco, 216 p.

Staub, Vincent (2006) La Libye et les migrations subsahariennes, Paris, L'Harmattan, 120 p.

Weber, Florence (2009) Manuel de l'ethnographe, Paris, Presses universitaires de France, $334 \mathrm{p}$.

\section{NOTES}

1. Mouammar Kadhafi est populairement connu comme le Guide, à cause de son autoproclamation comme leader de la révolution libyenne et aussi de plusieurs mouvements internationalistes, anti-impérialistes et révolutionnaires qu'il mène au niveau mondial.

2. Le peuple Songhay-Zarma se consacre traditionnellement à l'agriculture sur les pourtours du fleuve Niger. Il a aussi hérité, de part sa relation passée avec la colonie, des postes fournis par l'administration nigérienne.

3. Bien que l'on constate l'existence de combattants noirs affiliés à des milices de Kadhafi pour des motivations purement financières, les chiffres sont en réalité "marginaux", d'après Moisseron et Belalimat (2012). 
4. Même si les mouvements s'intègrent dans une migration circulaire - aller, retour, aller... - dans ce cas, il s'agit de "retours involontaires ", selon les termes utilisés par Kleist (2017) dans son ouvrage sur les personnes rentrées de Libye au Ghana. Ce sont des retours imprévus, précaires et forcés par la situation de conflit et les ultimatums des rebelles libyens envers les Noirs Africains. 5. Les chiffres varient selon la source, mais les estimations notent qu'Agadez et Tahoua sont les régions les plus affectées par ces retours tandis que la capitale reçoit moins de migrants, environ 5 000-10 000 personnes, selon des témoignages recueillis dans notre travail de terrain.

6. En 2012, la région de l'Azawad a connu une courte indépendance (déclarée unilatéralement), suite à la convergence de factions islamistes telles que Ansar Dine et le Mouvement pour l'Unification et le Jihad en Afrique de l'Ouest (MUJAO), avec les groupes Touaregs tel que le Mouvement National pour la Libération de l'Azawad (MNLA). En février 2013, la région rejoint le Mali. Mais l'insurrection armée au nord du Mali, suivie d'un coup d'État à Bamako, ont entrainé un conflit civil dans la région - conflit auquel les armées française et tchadienne ont pris part.

7. Les attaques de la secte islamiste s'étendent au cours de 2015 aux pays voisins du Nigeria, notamment le Niger, le Tchad et le Cameroun.

8. Le terme annassara fait référence au blanc/occidental. C'est une expression locale qui exemplifie la construction de l'Autre, assimilant la personne occidentale au « colonisateur ». Cela correspond à d'autres mots trouvés dans d'autres pays comme tubab au Senegal ou vazaha à Madagascar, entre autres.

9. Nous nous sommes malgré tout rendu à Agadez d'une manière ponctuelle pour observer et comprendre les explications liées au parcours migratoire du groupe qui fait l'objet de notre étude.

10. Notre première expérience au Niger fût en tant que stagiaire de l'Agence Espagnole de Coopération Internationale pour le Développement (AECID) en 2011. Ce travail nous a permis de nous lier avec des acteurs de la migration et nous a également servi pour former une première vision critique du secteur du développement, conçu comme un vecteur indispensable au système capitaliste en vigueur.

11. Nous concevons la "modernité », en tant que "développement qui conduit à une société économiquement entrepreneuse, technologiquement sophistiquée et politiquement ouverte ", suivant la définition de Chabal et Daloz (1999).

12. Après la chute de Kadhafi, le Niger, en tant que principal pays de transit, est devenu le nouveau gendarme de la migration irrégulière depuis l'Afrique sub-saharienne vers l'Europe. Il s'est érigé comme État prioritaire du Fonds Fiduciaire pour les Migrations de l'Union européenne par lequel le gouvernement nigérien reçoit des nombreux sommes d'argent pour contenir les flux de personnes, en contribuant à édifier une nouvelle frontière de la Forteresse Europe (Brachet, 2018).

13. Dans ce cas, le concept s'utilise pour rendre visible notre accueil dans la communauté nigérienne. Cependant, le terme s'utilise souvent pour faire référence aux migrants nigériens résidents en Europe et qui ne sont jamais revenus pour visiter la famille. Il s'agit d'une critique sur leur « acculturation » présupposé.

14. La Déclaration universelle des Droits de l'Homme déclare dans son article 13 que «toute personne a le droit de circuler librement et de choisir sa résidence à l'intérieur d'un Etat et toute personne a le droit de quitter tout pays, y compris le sien, et de revenir dans son pays ». 


\section{RÉSUMÉS}

La seconde moitié du XXème siècle voit la Libye apparaitre comme un Eldorado pour des milliers de migrants sub-sahariens attirés par des perspectives d'emploi et par la rhétorique panafricaine de Mouammar Kadhafi. Alors que la mal nommée "crise des réfugiés " affecte les régions du pourtour méditerranéen, la Libye est donc depuis longtemps un pays de destination. L'expression haoussa Libya Kaman Turaï, que nous pouvons traduire par « La Libye comme l'Europe », résume l'idée que le pays pourrait satisfaire les besoins économiques et sociaux des nouveaux venus au même titre que l'Europe. La guerre en Libye et la chute de Kadhafi en 2011 entrainent le retour forcé d'environ 300000 ressortissants nigériens vers leur pays d'origine. Ces derniers sont recherchés et accusés de complicité avec le régime khadafiste, voire même qualifiés de "mercenaires », par les rebelles du Conseil National de Transition (CNT) et une grande partie de la société libyenne. Notre ethnographie à Niamey, capitale du Niger, met en évidence les conditions de vie de ce groupe, dont les individus modifient leur rôle social et familial après l'échec de leur projet migratoire. L'instabilité de la région d'une part, et les contraintes financières que nous rencontrons lors du projet de recherche d'autre part, restreignent l'enquête à l'espace urbain. Néanmoins, ce sont ces contraintes qui ont permis d'exposer un phénomène jusqu'alors ignoré par la production scientifique, plutôt focalisée sur le milieu rural (Nabara, 2014). À travers des méthodes ethnographiques -observation participante et conversations informelles- dans des fadas, principaux espaces de sociabilité masculine du pays, nous révélons les obstacles d'un terrain vulnérable à travers un virage réflexif qui nous emmène à la déconstruction et à une recherche honnête, empathique et engagée (Guber, 1991).

\section{INDEX}

Index géographique : Niger, Libye

Mots-clés : projet migratoire, retour, ethnographie, espace urbain

\section{AUTEUR}

\section{ORIOL PUIG}

Docteur en Anthropologie Sociale, Groupe d'Étude des Sociétés Africaines (GESA) / Université de Barcelone uri.puigc@gmail.com 